\title{
SYNTHESIS, ANTIOXIDANT AND ANTICANCER ACTIVITY OF NEW QUINOLINE-[1, 2, 4]-TRIAZOLE HYBRIDS
}

\author{
Hemant Hegde ${ }^{1}$, Santosh L. Gaonkar ${ }^{1}$, Naveen P. Badiger ${ }^{2}$ \\ and Nitinkumar S. Shetty ${ }^{*}$ \\ ${ }^{1}$ Department of Chemistry, Manipal Institute of Technology, Manipal Academy of Higher \\ Education, Manipal-576104, (Karnataka) India \\ ${ }^{2}$ P.G. Dept. of Chemistry, JSS College, Vidyagiri, Dharwad-580004, (Karnataka) India \\ *E-mail: nitin.shetty@manipal.edu
}

\begin{abstract}
A set of quinoline coupled triazole derivatives was synthesized using substituted quinoline hydrazide and isothiocyanate derivatives. The structures of the synthesized molecules were confirmed using spectral techniques like FTIR, ${ }^{1} \mathrm{H} \&{ }^{13} \mathrm{C}$ NMR spectroscopy. Novel triazole thione derivatives were subjected to anticancer and antioxidant activity. The studies revealed the moderate anticancer activity of the molecules but have shown potent antioxidant activity.

Keywords: Quinoline-[1, 2, 4]-triazole, Molecular Hybrids, Anticancer Activity, Antioxidant Activity.
\end{abstract}

(C) RASĀYAN. All rights reserved

\section{INTRODUCTION}

The 1, 2, 4-triazoles are the vital units of an exigent class of heterocycles. They offer outstretched pharmacological benefits as antimicrobial, anticancer, antioxidant and several other applications. ${ }^{1-3}$ In the recent progress in medicinal chemistry, the synthesis of various 3-mercapto-1, 2, 4- triazole derivatives have gathered a considerable attraction owing to their significance in the field of medicinal chemistry. ${ }^{4-8}$ The biological activities exhibited by the 3-mercapto-1, 2, 4- triazoles is found to be because of the presence of $=\mathrm{N}-\mathrm{C}-\mathrm{S}$ moiety. ${ }^{9}$

On the other hand, quinolines are another important heterocyclic member that grabbed the attention of many researchers owing to its wide spectrum of pharmaceutical application. ${ }^{10-13}$ In the present work, 5-chloro-8hydroxy quinoline is derivatized which is an analog of a clioquinol (a broad-spectrum antibacterial and antifungal agent). Structural modification of the basic skeleton would help in the identification of new lead molecules to fight against life-threatening diseases like cancer ${ }^{14}$ Not much work has been done on the development of 1, 2, 4-triazole-quinoline hybrid. Shaker ${ }^{15}$ reviewed the most important procedures for the synthesis of 3-mercapto-1, 2, 4- triazoles. It is reported that the 3-mercapto-1, 2, 4- triazole has proved to be a rich source of various heterocyclic compounds. Fascinated by the biological profiles of triazoles and quinolines, we have designed and synthesized a new series of hybrid molecules that would produce a synergistic effect which also helps in the development of potential therapeutic agents. Recent findings reported that the antioxidants help in reducing the metastasis by reducing reactive oxygen species in cancer cells. ${ }^{16}$ This prompted us to test the newly synthesized compounds for their in vitro anticancer and antioxidant activity.

\section{EXPERIMENTAL}

Chemicals were purchased commercially and were used as such without any purification. The reaction progress was monitored with the help of Thin-layer chromatography (TLC). Aluminum sheets precoated with alichrosep silica gel-60/UV 254 was used as a stationary phase with iodine and UV light as visualizing agents. The melting point was checked using Thiele's tube in an open capillary tube. Functional group identification was done by FTIR spectra which were recorded on Shimadzu Infrared spectrometer (8400s)

Rasayan J. Chem., 13(3), 1744-1749(2020)

http://dx.doi.org/10.31788/RJC.2020.1335669

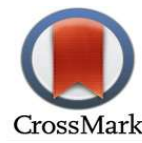


RASĀYAN J. Chem.

Vol. 13 | No. 3 |1744-1749| July - September | 2020

using $\mathrm{KBr}$ as background. ${ }^{1} \mathrm{H}$ and ${ }^{13} \mathrm{C}$ NMR spectra were recorded on Bruker NMR-400 MHz and $100 \mathrm{MHz}$ respectively with TMS as an internal standard. Elemental analysis was performed by using Perkin Elmer (2400) CHN elemental analyzer.

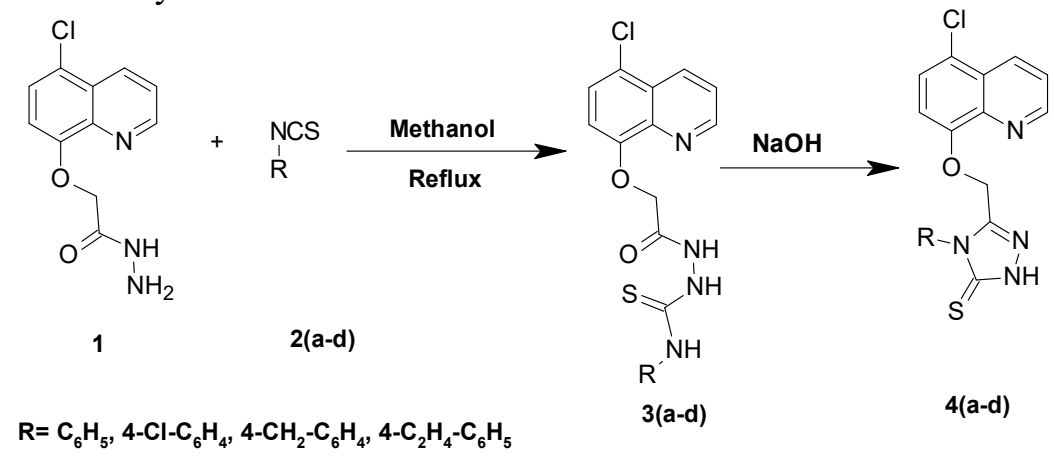

Scheme-1: Synthesis of Quinolinyl Triazole Hybrids

\section{Synthesis of Quinoline Hydrazide Derivative, (1)}

5-Chloro-quinolin-8-yloxy-acetohydrazide (1) was prepared by reacting corresponding esters $(1 \mathrm{mmol})$ with hydrazine hydrate, $99 \%(1.5 \mathrm{mmol})$ in ethanol $(2 \mathrm{~mL})$. Solid thus formed was filtered, dried, and used directly for the next step without further purification (recrystallization). ${ }^{17,18}$

\section{2-[(5-chloroquinolin-8-yl)oxy]acetohydrazide (1)}

Yield, $95 \%$, m. p. $118-120^{\circ} \mathrm{C}$; FTIR (KBr): $v_{\max }=3319(\mathrm{~N}-\mathrm{H}), 3041(\mathrm{Ar}-\mathrm{H}), 2936\left(\mathrm{CH}_{2}\right), 1672(\mathrm{C}=\mathrm{O})$, $1616(\mathrm{C}=\mathrm{N}), 1506(\mathrm{C}=\mathrm{C}), 777(\mathrm{Ar}-\mathrm{C}-\mathrm{Cl}) \mathrm{cm}^{-1} .{ }^{1} \mathrm{H}$ NMR $\left(400 \mathrm{MHz}, \mathrm{DMSO}-\mathrm{d}_{6}\right) \delta: 8.87(\mathrm{~d}, 1 \mathrm{H}, \mathrm{J}=3.4 \mathrm{~Hz}$, quinoline ring- $\mathrm{H}), 8.14(\mathrm{~d}, 1 \mathrm{H}, \mathrm{J}=8.4 \mathrm{~Hz}$, quinoline ring- $\mathrm{H}), 7.68-6.84(\mathrm{~m}, 11 \mathrm{H}, \mathrm{Ar}-\mathrm{H}), 4.76\left(\mathrm{~s}, 2 \mathrm{H}, \mathrm{OCH}_{2}\right)$, 4.39 (s, 2H, N-H) ppm. ${ }^{13} \mathrm{C}$ NMR (100 MHz, DMSO-d 6 ) $\delta 68.4,111.8,122.2,123.6,126.7,127.2,132.8$, 140.8, 150.5, 153.8, 166.9 ppm. Elemental analysis, Anal. calctd. for $\mathrm{C}_{11} \mathrm{H}_{10} \mathrm{ClN}_{3} \mathrm{O}_{2}: \mathrm{C}, 52.47 ; \mathrm{H}, 3.96 ; \mathrm{N}$, 16.74. Found: C, $52.50 ; \mathrm{H}, 4.01 ; \mathrm{N}, 16.70 \% .^{18}$

\section{Synthesis of Quinolinyl Carbothioamide 3(a-d)}

An equimolar mixture of quinoline hydrazide $1(2 \mathrm{mmol})$ and aryl-substituted isothiocyanate, 2(a-d) was refluxed in methanol $(5 \mathrm{~mL}$ ). On completion of the reaction (as confirmed by TLC), reaction content was cooled and filtered the solid thus formed. The crude product was recrystallized by using methanol to afford pure quinolinyl carbothioamide 3(a-d).

\section{2-(2-((5-chloroquinolin-8-yl)oxy)acetyl)-N-phenylhydrazine-1-carbothioamide, (3a)}

Yield, $91 \%$, m. p. 146-148 ${ }^{\circ} \mathrm{C}$; FTIR (KBr): $v_{\max }=3319,3025,1682,1552,1309,1238,781 \mathrm{~cm}^{-1} ;{ }^{1} \mathrm{H}$ NMR (400 MHz, DMSO-d 6 ), $\delta: 10.28(1 \mathrm{H}, \mathrm{s}, \mathrm{NH}), 9.76(1 \mathrm{H}, \mathrm{s}, \mathrm{NH}), 9.64(1 \mathrm{H}, \mathrm{s}, \mathrm{NH}), 9-9.02(\mathrm{~d}, 1 \mathrm{H}, \mathrm{J}=4 \mathrm{~Hz}$, Quinoline ring $\mathrm{H}), 8.54-8.56(\mathrm{~d}, 1 \mathrm{H}, \mathrm{J}=8 \mathrm{~Hz}$, Quinoline ring- $\mathrm{H}), 7.71-7.78(2 \mathrm{H}, \mathrm{d}$, Quinoline ring- $\mathrm{H}, \mathrm{J}=$ $8 \mathrm{~Hz}), 7.67-7.69(2 \mathrm{H}, \mathrm{d}$, Quinoline ring- $\mathrm{H}, \mathrm{J}=12 \mathrm{~Hz}), 7.38-7.42(1 \mathrm{H}, \mathrm{m}$, Quinoline ring-H), 7.30-7.34 $(3 \mathrm{H}, \mathrm{m}, \mathrm{Ar}-\mathrm{H}), 4.86\left(2 \mathrm{H}, \mathrm{s}, \mathrm{CH}_{2}\right) \mathrm{ppm} ;{ }^{13} \mathrm{C}$ NMR $\left(100 \mathrm{MHz}, \mathrm{DMSO}-\mathrm{d}_{6}\right), \delta: 66.7,113.1,117.3,120.5$, $122.9,123.4,125.8,126.2,130.4,136.7,142.7,151.7,155.3$ ppm; Anal. Calctd. for $\mathrm{C}_{18} \mathrm{H}_{15} \mathrm{ClN}_{4} \mathrm{O}_{2} \mathrm{~S}: \mathrm{C}$, $55.89 ; \mathrm{H}, 3.91 ; \mathrm{N}, 14.48$. Found: C, 56.02; H, 3.95; N, 14.44 .

N-(4-Chlorophenyl)-2-(2-((5-chloroquinolin-8-yl)oxy)acetyl)hydrazine-1-carbothioamide, (3b) Yield, $87 \%$, m. p. $152-153^{\circ} \mathrm{C}$; FTIR (KBr): $v_{\max }=3209,3021,1689,1533,1311,1240,783 \mathrm{~cm}^{-1} ;{ }^{1} \mathrm{H}$ NMR $\left(400 \mathrm{MHz}, \mathrm{DMSO}-\mathrm{d}_{6}\right), \delta: 10.40(1 \mathrm{H}, \mathrm{s}, \mathrm{NH}), 9.83(1 \mathrm{H}, \mathrm{s}, \mathrm{NH}), 9.81(1 \mathrm{H}, \mathrm{s}, \mathrm{NH}), 9.0-9.01(\mathrm{~d}, 1 \mathrm{H}, \mathrm{J}=4$ $\mathrm{Hz}$, Quinoline ring $\mathrm{H}), 8.54-8.56(\mathrm{~d}, 1 \mathrm{H}, \mathrm{J}=8 \mathrm{~Hz}$, Quinoline ring- $\mathrm{H}), 7.71-7.77(2 \mathrm{H}, \mathrm{d}$, Quinoline ring- $\mathrm{H}$, $\mathrm{J}=8 \mathrm{~Hz}), 7.47-7.49(2 \mathrm{H}, \mathrm{d}$, Quinoline ring- $\mathrm{H}, \mathrm{J}=12 \mathrm{~Hz}), 7.40-7.43(1 \mathrm{H}, \mathrm{m}$, Quinoline ring-H), 7.28-7.30 $(2 \mathrm{H}, \mathrm{d}, \mathrm{Ar}-\mathrm{H}, \mathrm{J}=8 \mathrm{~Hz}), 4.93\left(2 \mathrm{H}, \mathrm{s}, \mathrm{CH}_{2}\right) \mathrm{ppm} ;{ }^{13} \mathrm{C} \mathrm{NMR}\left(100 \mathrm{MHz}, \mathrm{DMSO}-\mathrm{d}_{6}\right.$ ) $, \delta: 68.04,113.9,117.6$, 
N-Benzyl-2-(2-((5-chloroquinolin-8-yl)oxy)acetyl)hydrazine-1-carbothioamide, (3c)

Yield, $82 \%$, m. p. $150-152^{\circ} \mathrm{C}$; FTIR (KBr): $v_{\max }=3209,3021,1689,1533,1311,1240,783 \mathrm{~cm}^{-1} ;{ }^{1} \mathrm{H}$ NMR $\left(400 \mathrm{MHz}, \mathrm{DMSO}-\mathrm{d}_{6}\right), \delta: 10.40(1 \mathrm{H}, \mathrm{s}, \mathrm{NH}), 9.83(1 \mathrm{H}, \mathrm{s}, \mathrm{NH}), 9.81(1 \mathrm{H}, \mathrm{s}, \mathrm{NH}), 9.0-9.01(\mathrm{~d}, 1 \mathrm{H}, \mathrm{J}=4$ $\mathrm{Hz}$, Quinoline ring $\mathrm{H}), 8.54-8.56(\mathrm{~d}, 1 \mathrm{H}, \mathrm{J}=8 \mathrm{~Hz}$, Quinoline ring-H), 7.71-7.77 $(2 \mathrm{H}, \mathrm{d}$, Quinoline ring- $\mathrm{H}$, $\mathrm{J}=8 \mathrm{~Hz}), 7.47-7.49(2 \mathrm{H}, \mathrm{d}$, Quinoline ring- $\mathrm{H}, \mathrm{J}=12 \mathrm{~Hz}), 7.40-7.43(1 \mathrm{H}, \mathrm{m}$, Quinoline ring-H), 7.28-7.30 $(2 \mathrm{H}, \mathrm{d}, \mathrm{Ar}-\mathrm{H}, \mathrm{J}=8 \mathrm{~Hz}), 4.93\left(2 \mathrm{H}, \mathrm{s}, \mathrm{CH}_{2}\right) \mathrm{ppm} ;{ }^{13} \mathrm{C} \mathrm{NMR}\left(100 \mathrm{MHz}, \mathrm{DMSO}-\mathrm{d}_{6}\right.$ ), $\delta: 35.41,68.01,112.3$, 113.1, 123.4, 126.3, 126.8, 127.3, 129.1, 129.7, 132.6, 139.6, 140.9, 150.6, 154.2 ppm; Anal. Calcd. for $\mathrm{C}_{19} \mathrm{H}_{17} \mathrm{ClN}_{4} \mathrm{O}_{2} \mathrm{~S}: \mathrm{C}, 56.93 ; \mathrm{H}, 4.27 ; \mathrm{N}, 13.98$. Found: C, 57.01; H, 4.32; N, 14.06.

2-(2-((5-Chloroquinolin-8-yl)oxy)acetyl)-N-phenethylhydrazine-1-carbothioamide, (3d)

Yield, $95 \%$, m. p. $148-150{ }^{\circ} \mathrm{C}$; FTIR (KBr): $v_{\max }=3228,1681,1556,1504,1311,783 \mathrm{~cm}^{-1} ;{ }^{1} \mathrm{H}$ NMR (400 MHz, DMSO-d 6 ), $\delta: 9.0(1 \mathrm{H}, \mathrm{s}, \mathrm{NH}), 8.54-8.56(\mathrm{~d}, 1 \mathrm{H}, \mathrm{J}=8 \mathrm{~Hz}$, Quinoline ring-H), $8.19(1 \mathrm{H}, \mathrm{s}, \mathrm{NH})$, 7.71-7.77 (2H, d, Quinoline ring-H, J = 8 Hz), 7.47-7.49 (2H, d, Ar-H, J = $12 \mathrm{~Hz}), 7.40-7.43(1 \mathrm{H}, \mathrm{m}$, Quinoline ring- $\mathrm{H}), 7.19-7.32(8 \mathrm{H}, \mathrm{m}, \mathrm{Ar}-\mathrm{H}), 4.89\left(2 \mathrm{H}, \mathrm{s}, \mathrm{CH}_{2}\right), 3.62-3.67\left(2 \mathrm{H}, \mathrm{s}, \mathrm{CH}_{2}\right), 2.80-2.84(2 \mathrm{H}, \mathrm{m}$, $\left.\mathrm{CH}_{2}\right) \mathrm{ppm} ;{ }^{13} \mathrm{C}$ NMR $\left(100 \mathrm{MHz}, \mathrm{DMSO}-\mathrm{d}_{6}\right), \delta: 35.35,40.06,68.0,112.3,112.9,123.6,126.6,126.7$, 127.1, 128.8, 129.0, 132.8, 139.6, 140.7, $150.5,153.8$ ppm; Anal. Calcd. for $\mathrm{C}_{20} \mathrm{H}_{19} \mathrm{ClN}_{4} \mathrm{O}_{2} \mathrm{~S}: \mathrm{C}, 57.90 ; \mathrm{H}$, 4.62; N, 13.50. Found: C, 57.98; H, 4.59; N, 13.38 .

\section{Synthesis of Quinolinyl Triazole 4(a-d)}

Quinoline carbothioamide 3(a-d) $(1 \mathrm{mmol})$ with $5 \% \mathrm{NaOH}$ (aqueous) at $70^{\circ} \mathrm{C}$ for $1 \mathrm{~h}$ to form the titled compounds 4(a-d). Progress of the reaction was monitored by using thin-layer chromatography by taking toluene: ethyl acetate; 2:1 respectively as mobile phase. Cooled and acidified with $6 \mathrm{~N} \mathrm{HCl}$ and crystallized from ethanol to afford pure quinolinyl triazole derivatives $\mathbf{4}(\mathbf{a}-\mathbf{d})$. The crude product was recrystallized using methanol.

5-((5-Chloroquinolin-8-yl)oxy)methyl)-4-phenyl-2,4-dihydro-3H-1,2,4-triazole-3-thione (4a) Yield: $78 \%$, m.p. $218-220^{\circ} \mathrm{C}$, FTIR (KBr): $v_{\max }=3436,3045,1612,1589,1467,783 \mathrm{~cm}^{-1}$; ${ }^{1} \mathrm{H}$ NMR $(400$ MHz, DMSO-d 6 ), $\delta: ~: 14.09(\mathrm{~s}, 1 \mathrm{H},-\mathrm{NH}), 8.54-9.01(\mathrm{~m}, 2 \mathrm{H}$, quinoline-H), 8.50-8.51 (d, $1 \mathrm{H}$, quinoline- $\mathrm{H}$, $\mathrm{J}=4 \mathrm{~Hz}), 7.76-7.77(\mathrm{~d}, 1 \mathrm{H}$, quinoline- $\mathrm{H}, \mathrm{J}=4 \mathrm{~Hz}), 7.22-7.75(\mathrm{~m}, 5 \mathrm{H}, \mathrm{Ar}-\mathrm{H}), 5.22\left(\mathrm{~s}, 2 \mathrm{H}, \mathrm{O}-\mathrm{CH}_{2}\right) \mathrm{ppm}$; ${ }^{13} \mathrm{C}-\mathrm{NMR}\left(100 \mathrm{MHz}, \mathrm{DMSO}-\mathrm{d}_{6}\right) \delta$ : 61.91, 112.4, 115.8, 116.3, 123.5, 123.9, 126.8, 130.3, 130.8, 131.5, 133.0, 140.8, 148.3, 150.9, 153.2, 161.4, 163.9, 169.4 ppm; Anal. Calctd for $\mathrm{C}_{18} \mathrm{H}_{13} \mathrm{ClN}_{4} \mathrm{OS}$ : C, 58.62; $\mathrm{H}$, $3.55 ; \mathrm{N}, 15.19$. Found: C, 58.58; H, 3.62; N, 15.24.

\section{4-(4-Chlorophenyl)-5-(((5-chloroquinolin-8-yl)oxy)methyl)-2,4-dihydro-3H-1,2,4-triazole-3- thione $(4 b)$}

Yield: $87 \%$, m. p. $237-238^{\circ} \mathrm{C}$, FTIR (KBr): $v_{\max }=3436,3045,1612,1589,1467,783 \mathrm{~cm}^{-1} ;{ }^{1} \mathrm{H}$ NMR $(400$ MHz, DMSO-d 6 ), $\delta: 14.13(\mathrm{~s}, 1 \mathrm{H},-\mathrm{NH}), 8.51-8.97(\mathrm{~m}, 2 \mathrm{H}$, quinoline-H), 8.49-8.5 (d, $1 \mathrm{H}$, quinoline- $\mathrm{H}, \mathrm{J}$ $=1.6 \mathrm{~Hz}), 7.72-7.73(\mathrm{~d}, 1 \mathrm{H}$, quinoline- $\mathrm{H}, \mathrm{J}=4 \mathrm{~Hz}), 7.20-7.67(\mathrm{~m}, 4 \mathrm{H}, \mathrm{Ar}-\mathrm{H}), 5.22\left(\mathrm{~s}, 2 \mathrm{H}, \mathrm{O}-\mathrm{CH}_{2}\right) \mathrm{ppm} ;{ }^{13} \mathrm{C}-$ NMR (100 MHz, DMSO-d 6 ) $\delta: 61.96,112.6,116.2,116.5,123.3,123.6,127.1,129.9,131.0,131.1,132.8$, 140.7, 148.4, 150.6, 152.7, 161.3, 163.7, 169.2 ppm; Anal. Calcd for $\mathrm{C}_{18} \mathrm{H}_{12} \mathrm{Cl}_{2} \mathrm{~N}_{4} \mathrm{OS}$ (\%): C 53.61, H 3.0, N 13.89; Found: C 53.58, H 2.92, N 14.01.

\section{4-Benzyl-5-(((5-chloroquinolin-8-yl)oxy)methyl)-2,4-dihydro-3H-1,2,4-triazole-3-thione (4c)}

Yield: $71 \%$, m. p. $230-232{ }^{\circ} \mathrm{C}$, FTIR (KBr): $v_{\max }=3428,3052,1609,1593,1462,785 \mathrm{~cm}^{-1},{ }^{1} \mathrm{H}$ NMR (400 MHz, DMSO-d 6$), \delta: 14.09(\mathrm{~s}, 1 \mathrm{H},-\mathrm{NH}), 8.55-8.94(\mathrm{~m}, 2 \mathrm{H}$, quinoline-H), 8.53-8.54 (d, $1 \mathrm{H}$, quinoline- $\mathrm{H}$, $\mathrm{J}=1.6 \mathrm{~Hz}), 7.75-7.76(\mathrm{~d}, 1 \mathrm{H}$, quinoline- $\mathrm{H}, \mathrm{J}=4 \mathrm{~Hz}), 7.23-7.71(\mathrm{~m}, 4 \mathrm{H}, \mathrm{Ar}-\mathrm{H}), 5.23\left(\mathrm{~s}, 2 \mathrm{H}, \mathrm{O}-\mathrm{CH}_{2}\right) \mathrm{ppm}$; ${ }^{13} \mathrm{C}-\mathrm{NMR}\left(100 \mathrm{MHz}, \mathrm{DMSO}-\mathrm{d}_{6}\right)$ $\delta: 61.8,112.4,116.2,116.5,123.3,123.6,127.1,129.9,131.0,131.1$, 132.8, 140.7, 148.4, 150.6, 152.7, 161.3, 163.7, $169.2 \mathrm{ppm}$; Anal. Calcd for $\mathrm{C}_{18} \mathrm{H}_{12} \mathrm{Cl}_{2} \mathrm{~N}_{4} \mathrm{OS}$ (\%): C 53.61, H 3.0, N 13.89; Found: C 53.58, H 3.05, N 13.95 
RASĀYAN J. Chem.

Vol. 13 | No. 3 |1744-1749| July - September | 2020

5-(((5-Chloroquinolin-8-yl)oxy)methyl)-4-phenethyl-2,4-dihydro-3H-1,2,4-triazole-3-thione Yield: $73 \%$, m. p. $224-226^{\circ} \mathrm{C}$, FTIR (KBr): $v_{\max }=3432,3054,1616,1591,1462,788 \mathrm{~cm}^{-1} ;{ }^{1} \mathrm{H}$ NMR (400 MHz, DMSO-d $\left.{ }_{6}\right), \delta: 14.11(\mathrm{~s}, 1 \mathrm{H},-\mathrm{NH}), 8.50-9.01(\mathrm{~m}, 2 \mathrm{H}$, quinoline-H), 8.51-8.514 (d, $1 \mathrm{H}$, quinoline- $\mathrm{H}$, $\mathrm{J}=1.6 \mathrm{~Hz}), 7.69-7.70(\mathrm{~d}, 1 \mathrm{H}$, quinoline- $\mathrm{H}, \mathrm{J}=4 \mathrm{~Hz}), 7.19-7.62(\mathrm{~m}, 4 \mathrm{H}, \mathrm{Ar}-\mathrm{H}), 5.22\left(\mathrm{~s}, 2 \mathrm{H}, \mathrm{O}-\mathrm{CH}_{2}\right), 3.59-$ $3.62\left(\mathrm{~m}, 2 \mathrm{H}, \mathrm{CH}_{2}\right), 2.68-2.70\left(\mathrm{~m}, 2 \mathrm{H}, \mathrm{CH}_{2}\right) \mathrm{ppm} ;{ }^{13} \mathrm{C}-\mathrm{NMR}\left(100 \mathrm{MHz}, \mathrm{DMSO}-\mathrm{d}_{6}\right) \delta: 34.37,40.6,61.9$, $112.4,116.2$, 117.2, 124.1, 125.1, 126.8, 130.4, 131.5, 132.2, 133.0, 140.8, 148.7, 150.9, 153.4, 161.5, 164.2, 169.5 ppm; Anal. Calcd for $\mathrm{C}_{20} \mathrm{H}_{17} \mathrm{ClN}_{4} \mathrm{OS}$ : C, 60.53; H, 4.32; N, 14.12; Found: C, 60.61; H, 4.37; $\mathrm{N}, 14.08$.

\section{RESULTS AND DISCUSSION}

The target compounds $\mathbf{4}(\mathbf{a}-\mathbf{d})$ were synthesized by the base-catalyzed cyclization of carbothioamide derivatives 3(a-d). The quinolinyl carbothioamides were formed by the reaction between quinoline hydrazide and substituted aryl isothiocyanates 2(a-d). The starting material quinoline hydrazide was prepared by reacting 5-chloro-8-hydroxy quinolinol with ethyl chloroacetate resulted in the formation of aryloxy ester of quinolinol. This on further reaction with hydrazine hydrate gave corresponding acetohydrazide derivative (1). All the molecules were tested for their antioxidant and anticancer activity. The spectral analysis of starting material quinoline hydrazide derivative has shown characteristic stretching vibration at $1678 \mathrm{~cm}^{-1}$ and $3319 \mathrm{~cm}^{-1}$ corresponds to $\mathrm{C}=\mathrm{O}$ and $\mathrm{NH}_{2}$ group respectively. It was further confirmed by the ${ }^{1} \mathrm{H}$ and ${ }^{13} \mathrm{C}$ NMR characterization. Proton NMR studies show the signal at $\delta 4.39$ and 4.76 ppm corresponds to $\mathrm{N}-\mathrm{H}$ and $-\mathrm{CH}_{2}$ protons. On reaction with isothiocyanates 2(a-d), the compound $\mathbf{1}$ forms corresponding carbothioamide derivatives 3(a-d) which is evident from the NMR signal where there is an absence of $\mathrm{NH}_{2}$ proton and signal corresponds to $-\mathrm{OCH}_{2}$ is remained in the spectrum with the slight shift in the region of $\delta 4.7-4.9 \mathrm{ppm}$. Further, the compounds 3(a-d) were cyclized to form the final product, quinoline triazole derivatives $\mathbf{4}(\mathbf{a}-\mathbf{d})$. The absence of the carbonyl group $(\mathrm{C}=\mathrm{O})$ in FTIR analysis confirmed the cyclization of carbothioamide derivatives.

\section{Antioxidant Activity of Synthesized Compounds DPPH Radical Scavenging Activity}

All the synthetic samples were dissolved in DMSO at $10 \mathrm{mM}$ stock concentration and $50 \mu \mathrm{L}$ was used for testing antioxidant activity at a final concentration of $250 \mu \mathrm{M}$. Out of eight samples, samples $\mathbf{3 a}, \mathbf{3 b}, \mathbf{3 c}$, and $\mathbf{4 c}$ did not show significant antioxidant activity against DPPH free radical as shown in Table-1. The remaining samples, which showed high antioxidant activity was tested at different concentrations to find out the $\mathrm{IC}_{50}$ values are represented in Table-2. Blanks were prepared for each sample by replacing DPPH with methanol and the dilutions of the samples were made in DMSO wherever required. All the experiments were repeated in triplicates.

Table-1: Antioxidant Activity of the Samples at $10 \mathrm{mM}$ Concentration (Sample Volume $50 \mu 1$ ).

\begin{tabular}{c|c|c}
\hline S. No. & Sample & Inhibition $\%$ \\
\hline 01 & 3a & 44.25 \\
\hline 02 & 3b & 51.885 \\
\hline 03 & 3c & 40.89 \\
\hline 04 & 3d & 92.932 \\
\hline 05 & $4 \mathrm{a}$ & 77.347 \\
\hline 06 & 4b & 82.756 \\
\hline 07 & 4c & 58.101 \\
\hline 08 & 4d & 92.412 \\
\hline
\end{tabular}

As shown in Table-1, compounds $\mathbf{3 d}$ and $\mathbf{4 d}$ have shown the best free radical scavenging behavior and are considered to be the potent antioxidants with the $92 \%$ inhibition. Which is also evident from the $\mathrm{IC}_{50}$ values (Table-2).

The compound 3d found to exhibit the most potent activity of all the synthesized molecules. The better activity of carbothioamide derivatives could be attributed to the open-chain structures with methylene 
RASĀYAN J. Chem.

Vol. 13 | No. 3 |1744-1749| July - September | 2020

spacer group as well as the presence of a polar group that can interact better with the enzyme that is inhibited. On the other hand, the titled quinoline-triazole hybrid molecules $\mathbf{4 ( a - d ) ~ h a v e ~ s h o w n ~ g o o d ~ t o ~}$ excellent antioxidant activity (on comparing with the standard) which can be accredited to the synergistic effect of two bioactive pharmacophore units in the same structure.

Table-2: Antioxidant Activity of the Samples represented in their $\mathrm{IC}_{50}$ Values

\begin{tabular}{c|c|c}
\hline S. No. & Synthetic Sample & $\begin{array}{c}\text { Antioxidant activity } \\
\left(\mathrm{IC}_{50} \mathrm{mM}\right)\end{array}$ \\
\hline 1 & $3 \mathrm{~d}$ & $0.096 \pm 0.005$ \\
\hline 2 & $4 \mathrm{a}$ & $1.003 \pm 0.049$ \\
\hline 3 & $4 \mathrm{~b}$ & $0.881 \pm 0.030$ \\
\hline 4 & $4 \mathrm{~d}$ & $0.238 \pm 0.018$ \\
\hline$*$ & Std (Ascorbic Acid) & $0.032 \pm 0.001$ \\
\hline
\end{tabular}

\section{In- vitro Anticancer Activity (MTT Assay)}

The stock solution was prepared by dissolving the synthesized compounds in DMSO as $10 \mathrm{mg} / \mathrm{mL}$ and was stored at $-20{ }^{\circ} \mathrm{C}$. The breast cancer cell line (MCF-7) was used for the in vitro anticancer study. Exponentially growing cells were collected from T- 25 tissue culture flasks and a stock cell suspension 1 X $10^{6}$ were prepared with respective media. Cells were seeded 5000 cells/well in sterile 96 -well flat-bottom tissue culture plate and allowed to attach for 24 hours. After 24 hours of incubation, cells were treated with $100 \mu \mathrm{l}$ of test compounds from the respective stock solution for 48 hours. To each well of the 96 well plates, $50 \mu \mathrm{l}$ of MTT reagent (Stock: $2 \mathrm{mg} / \mathrm{ml}$ in PBS) was added and incubated for 3 hours at $37^{\circ} \mathrm{C}$. The optical density (O.D) was measured by a well plate reader at a wavelength of $540 \mathrm{~nm}$. Percentage cell death of each compound was calculated by the formula below:

$$
\% \text { Cell Death }=\frac{\text { OD of Control }- \text { OD of Test }}{\text { OD of Control }} \times 100
$$

The results are tabulated as $\mathrm{IC}_{50}$ in Table-3.

Table-3: Anticancer Activity of the Samples represented in their $\mathrm{IC}_{50}$ Values.

\begin{tabular}{c|c|c}
\hline S. No. & Compound & $\mathrm{IC}_{50}(\mu \mathrm{M})$ \\
\hline 1 & $3 \mathrm{a}$ & $>200$ \\
\hline 2 & $3 \mathrm{~b}$ & $32.24 \pm 0.025$ \\
\hline 4 & $3 \mathrm{c}$ & $160.7 \pm 0.02$ \\
\hline 5 & $3 \mathrm{~d}$ & $59.5 \pm 0.026$ \\
\hline 6 & $4 \mathrm{a}$ & $63.26 \pm 0.003$ \\
\hline 8 & $4 \mathrm{~b}$ & $47.64 \pm 0.005$ \\
\hline 9 & $4 \mathrm{c}$ & $187.8 \pm 0.032$ \\
\hline 10 & $4 \mathrm{~d}$ & $117.6 \pm 0.015$ \\
\hline Standard & Doxorubicin & $0.82 \pm 0.001$ \\
\hline
\end{tabular}

As evident from Table- 3 compounds, $\mathbf{3 b}$ has shown best cytotoxicity against MCF-7 cell lines at $32.24 \mu \mathrm{M}$ concentration which is nearest to that of the standard doxorubicin $(0.82 \mu \mathrm{M})$. Among triazoles, the compound $\mathbf{4 b}$ having chlorophenyl ring as aromatic part in isothiocyanate has shown significant inhibition when compared to the other members of the series. The rest of all molecules of the series have exhibited poor inhibition activity against MCF-7 cell lines.

\section{CONCLUSION}

In conclusion, a new series of quinoline-[1, 2, 4]-triazole hybrid molecules were synthesized using various isothiocyanate derivatives and quinoline hydrazide. All molecules were tested for anticancer and antioxidant activities. Among them, 3d and 4d having phenethyl isothiocyanate substitution have shown significant antioxidant activity when compared to the standard ascorbic acid. Moderate growth inhibition is observed for MCF-7 cell lines. Interestingly, the observed results have shown that both the intermediate 
RASĀYAN J. Chem.

Vol. 13 | No. 3 |1744-1749| July - September | 2020

carbothioamide derivative 3(a-d) and the target compound 4(a-d) found to have significant pharmacological responses because of which it can be considered for the further development of new antioxidant and anticancer lead molecules.

\section{REFERENCES}

1. I. A. Al-Masoudi, Y. A. Al-Soud, N. J. Al-Salihi and N. A. Al-Masoudi, Chemistry of Heterocyclic Compound, 42, 1377(2006), DOI:10.1007/s10593-006-0255-3

2. P. P. Gadhave, N. S. Dighe, S. R. Pattan, P. Deotarse, D. S. Musmade and R. V. Shete, Annals of Biological Research, 1, 82(2010).

3. C. H. Zhou and Y. Wang, Current Medicinal Chemistry, 19, 239(2012), DOI: $10.2174 / 092986712803414213$

4. J. K. Sahu, S. Ganguly and A. Kaushik, Chinese Journal of Natural Medicine, 11, 456(2013), DOI: 10.1016/S1875-5364(13)60084-9

5. S. G. Küçükgüzel and P. Çıkla-Süzgün European Journal of Medicinal Chemistry, 97, 830(2015), DOI: 10.1016/j.ejmech.2014.11.033

6. Z. A. Kaplancikli, G. Turan-Zitouni, A. Ozdemir and G. Revial, European Journal of Medicinal Chemistry, 43, 155(2008), DOI:10.1016/j.ejmech.2007.03.019

7. K. Zamani, K. Faghihi, M. R. Sangi and J. Zolgharnein, Turkish Journal of Chemistry, 27, 119(2003).

8. S. G Umut, G. K. Nesrin, G. Ozgur, K. Yavuz, K. Ekrem, I. Samil, A. Goknur and O. Meral, Bioorganic and Medicinal Chemistry, 15, 5738(2007), DOI:10.1016/j.bmc.2007.12.012

9. B. S. Holla, N. K. Poorjary, S. B. Rao and M. K. Shivananda, European Journal of Medicinal Chemistry, 37, 511(2002), DOI:10.1016/s0223-5234(02)01358-2

10. S. Eswaran, A. V. Adhikari, I. H. Chowdhury, N. K. Pal and K. D. Thomas, European Journal of Medicinal Chemistry, 45, 3374(2010), DOI:10.1016/j.ejmech.2010.04.022

11. S. Kumar, S. Bawa and H. Gupta, Mini Reviews on Medicinal Chemistry, 9, 1648(2009), DOI: $10.2174 / 138955709791012247$

12. S. Eswaran, A. V. Adhikari and N. S. Shetty, European Journal of Medicinal Chemistry, 44, 4637 (2009), DOI:10.1016/j.ejmech.2009.06.031

13. R. S. Upadhayaya, J. K. Vandavasi, R. A. Kardile, S. V. Lahore, S. S. Dixit, H. S. Deokar, P. D. Shinde, M. P. Sarmah and J. Chattopadhyaya, European Journal of Medicinal Chemistry, 45, 1854(2010), DOI:10.1016/j.ejmech.2010.01.024

14. Z. Xiao, S. L. Morris-Natschke,and K. H. Lee, Medicinal Research Reviews, 36, 32(2016), DOI: $10.1002 / \mathrm{med} .21377$

15. R. M. Shaker, Arkivoc, IX, 59(2006), DOI:10.3998/ark.5550190.0013.101

16. M. Asadi-Samani, N. K. Farkhad, M. R. Mahmoudian-Sani and H. Shirzad, 2109, Antioxidants as a Double-Edged Sword in the Treatment of Cancer, Antioxidants, Emad Shalaby, IntechOpen, London, DOI: 10.5772 /intechopen. 85468

17. F. Hayat, A. Salahuddin, S. Umar and A. Azam, European Journal of Medicinal Chemistry, 45, 4669 (2010), DOI:10.1016/j.ejmech.2010.07.028

18. H. Hegde, C. Ahn, D. Shwetha, S. L. Gaonkar and N. S. Shetty, Journal of Korean Chemical Society, 61, 291(2017), DOI:10.5012/jkcs.2017.61.5.291

[RJC-5669/2020] 